\title{
A new strategy for repeated application of adenovirus based vectors: proof-of-concept in rhesus macaques challenged with SIVmac239
}

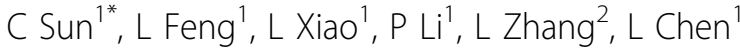 \\ From AIDS Vaccine 2012 \\ Boston, MA, USA. 9-12 September 2012
}

\section{Background}

It is well recognized that highly active antiretroviral therapy (HAART) can control HIV/AIDS and prolong patient's life. However, HAART is associated with drug toxicity, drug resistant, and patient's affordability. Therefore, developing an effective therapeutic vaccine that could induce HIV-specific immune responses may provide a solution to reduce the need of antiretroviral therapy. Adenovirus based vaccines have been extensively evaluated as a vaccine vehicle for HIV/AIDS and a variety infectious diseases, however, it has been a major concern that antiAd5 neutralizing antibodies in general population or after the first dose of immunization can hinder the its practical application. The idea of using less prevalent adenovirus serotypes lack of long-term safety record including oncogeneicity. Moreover, any adenovirus serotype will inevitablely induce neutralizing antibodies after one single use which render the strategy of using more serotypes infeasible.

\section{Methods}

In this study, we explored a novel "one-size-fits-all" strategy, namely AVIP (Adenoviral Vector Infected PBMC), to circumvent the attenuated efficacy of Ad-based vaccines due to anti-Ad immunity.

\section{Results}

We demonstrated that this AVIP strategy can elicit SIVspecific responses in both Ad5-seropositive and Ad5-seronegative macaques. Interestingly, comparable SIV-specific but weaker Ad5-specific responses were elicited in Ad5seronegative monkeys received AVIP immunization as compared to direct injection of Ad5-SIV vaccines. Then a cohort of Ad5-seropositive SIVmac239-infected monkeys received AVIP immunization with Ad5 vectors expressing SIVmac239 Gag, Pol and Env with HAART. After therapeutic immunization, SIV-specific response was induced in vaccinated monkeys whereas a slower responses were observed in non-vaccinated monkeys. The functional immunological profiles were also being detected, and we are also monitoring whether this immunological benefit would afford a virological benefit.

\section{Conclusion}

Our goal is to further develop the AVIP strategy as a simple but practically effective method for repeated delivery of Ad5-based vaccines in humans for HIV and other diseases.

\section{Author details}

${ }^{1}$ Guangzhou Institute and Biomedicine and Health (GIBH), Guangzhou, China. ${ }^{2}$ Comprehensive AIDS Research Center, Tsinghua University, Beijing, China.

Published: 13 September 2012

doi:10.1186/1742-4690-9-S2-P335

Cite this article as: Sun et al: A new strategy for repeated application of adenovirus based vectors: proof-of-concept in rhesus macaques challenged with SIVmac239. Retrovirology 2012 9(Suppl 2):P335.

${ }^{1}$ Guangzhou Institute and Biomedicine and Health (GIBH), Guangzhou, China Full list of author information is available at the end of the article

(c) 2012 Sun et al; licensee BioMed Central Ltd. This is an Open Access article distributed under the terms of the Creative Commons 